\title{
A CHARACTERISTIC INITIAL VALUE PROBLEM FOR A STRICTLY HYPERBOLIC SYSTEM
}

\author{
NEZAM IRANIPARAST
}

Received 6 August 2003

\begin{abstract}
Consider the system $A u_{t t}+C u_{x x}=f(x, t),(x, t) \in T$ for $u(x, t)$ in $\mathbb{R}^{2}$, where $A$ and $C$ are real constant $2 \times 2$ matrices, and $f$ is a continuous function in $\mathbb{R}^{2}$. We assume that $\operatorname{det} C \neq 0$ and that the system is strictly hyperbolic in the sense that there are four distinct characteristic curves $\Gamma_{i}, i=1, \ldots, 4$, in $x t$-plane whose gradients $\left(\xi_{1 i}, \xi_{2 i}\right)$ satisfy $\operatorname{det}\left[A \xi_{1 i}^{2}+\right.$ $\left.C \xi_{2 i}^{2}\right]=0$. We allow the characteristics of the system to be given by $d t / d x= \pm 1$ and $d t / d x=$ $\pm r, r \in(0,1)$. Under special conditions on the boundaries of the region $T=\{(x, t): 0 \leq t \leq$ $1,(-1+r+t) / r \leq x \leq(1+r-t) / r\}$, we will show that the system has a unique $C^{2}$ solution in $T$.
\end{abstract}

2000 Mathematics Subject Classification: 35L50, 35L20, 35C15.

1. Introduction. In the single equation case, Kal'menov [1] used separation of variables to explicitly find the eigenvalues and a complete set of eigenfunctions in $L^{2}\left(T_{1}\right)$ for

$$
\begin{gathered}
u_{t t}-u_{x x}=\lambda u, \quad(x, t) \in T_{1}, \\
u(x, 0)=0, \quad 0 \leq x \leq 2, \\
u(t, t)=u(1+t, 1-t), \quad 0 \leq t \leq 1,
\end{gathered}
$$

where

$$
T_{1}=\{(x, t): 0 \leq t \leq 1, t \leq x \leq 2-t\}
$$

is a triangular region bounded by two characteristics and the $x$-axis. In [3], Kreith generalized the result of Kal'menov [1] to the case where separation of variables was not necessarily possible, that is, the problem

$$
\begin{gathered}
u_{t t}-u_{x x}=\lambda p u, \quad(x, t) \in T_{1}, \\
u(x, 0)=0, \quad 0 \leq x \leq 2, \\
u(t, t)=u(1+t, 1-t), \quad 0 \leq t \leq 1,
\end{gathered}
$$

where $u \in \mathbb{R}$ and $p$ is a positive continuous function in $T_{1}$. In [3], Kreith used a symmetric Green's function to show the existence of the eigenvalues and a complete set of eigenfunctions in $L_{2}^{p}\left(T_{1}\right)$. His technique involved converting the eigenvalue problem (1.3) to an integral equation with a symmetric kernel. Both of these works were significant because problems (1.1) and (1.3) constituted selfadjoint boundary value problems 
for hyperbolic equations comparable to the ones for traditional elliptic equations. In addition, the boundary conditions in these problems imply $u(1,1)=0$. On the physical grounds, this means the string which was in equilibrium initially is again in equilibrium at a point at another time. In this context, one can think of the point $(1,1)$ as a generalized conjugate point for the initial condition $u(x, 0)=0$ [3].

In an attempt to extend Kreith's case to systems, we now consider

$$
A u_{t t}+C u_{x x}=f(x, t), \quad(x, t) \in T,
$$

where

$$
T=\left\{(x, t): 0 \leq t \leq 1, \frac{-1+r+t}{r} \leq x \leq \frac{1+r-t}{r}\right\}
$$

and apply similar but modified boundary conditions on the boundaries of $T$,

$$
\begin{aligned}
& u(x, 0)=0, \quad 1-\frac{1}{r} \leq x \leq 1+\frac{1}{r}, \quad 0<r<1, \\
& u\left(\frac{r-1+t}{r}, t\right)=u\left(1+\frac{t}{r}, 1-t\right)=g(r ; t), \quad 0<r<1,0 \leq t \leq 1 \text {. }
\end{aligned}
$$

The function $g$ is taken to be $C^{2}$ in $t$ for $t \in[0,1]$ for any $r \in(0,1)$, and its components $g_{1}$ and $g_{2}$ vanish monotonically to zero as $t$ goes to zero or one in $\left[0, t_{0}\right] \cup\left[t_{1}, 1\right]$ for some $t_{0}<t_{1}, t_{0}, t_{1} \in(0,1)$. For the sake of specificity, we assume that the constant matrices $A$ and $C$ with $\operatorname{det} C \neq 0$ are such that

$$
C^{-1} A=\left[\begin{array}{cc}
-1-d-r^{2} & \frac{-d-d^{2}-r^{2}-d r^{2}}{c} \\
c & d
\end{array}\right] .
$$

We note here that the boundary conditions (1.6) and (1.7) imply the compatibility condition $u(1,1)=0$, which in turn means that the system which was in equilibrium at time $t=0$ will come to rest at the point $x=1$ at the time $t=1$ again. Assumption (1.8) will guarantee the strict hyperbolicity [2] of system (1.4). In fact, let the polynomial $q$ be $q(\xi, \eta)=\operatorname{det}\left[A \xi^{2}+C \eta^{2}\right]$. Then, $q(1, m)=(\operatorname{det} C)\left(\operatorname{det}\left[C^{-1} A+m^{2} I\right]\right)$, where $I$ is the identity matrix and has four distinct roots $m= \pm 1$ and $m= \pm r$. If we let the equations of the characteristics be $t=\phi(x)$, then they will satisfy $d t / d x= \pm 1$ and $d t / d x= \pm r$. Accordingly, the characteristics of (1.4) are $\Gamma_{1}: t=x+k_{1}, \Gamma_{2}: t=-x+k_{2}, \Gamma_{3}: t=r x+k_{3}$, and $\Gamma_{4}: t=-r x+k_{4}$. We choose the characteristics $t=r x+1-r$ and $t=-r x+1+r$ in $x t$-plane, and form the triangular region $T$, described above, bounded by these lines and the $x$-axis. To find the points in condition (1.7), start at a point $((r-1+t) / r, t)$ on $t=r x+1-r$, and draw a line parallel to $t=-r x+1+r$. At the point of intersection of this line with the $x$-axis, draw a line parallel to $t=r x+1-r$ to meet the line $t=-r x+1+r$ at the point $(1+t / r, 1-t)$.

The original purpose of this study was to generalize the work in [3] to the case of a boundary value problem for a hyperbolic system which would be selfadjoint. But, after successfully defining the right domain $T$ and converting problem (1.4), (1.5), (1.6), and (1.7) to an integral equation over $T$, the kernel of the integral operator did not turn 
out to be symmetric. This precluded a statement similar to the one in [3] regarding the eigenfunctions and eigenvalues of (1.4), (1.5), (1.6), and (1.7) with $f(x, t)=\lambda p(x, t) u$. However, we were able to show, as we will explain in the sequel, that the problem does have a solution. The method is constructive and produces a solution which is $C^{2}$ and unique in $T$.

We mention further that characteristic boundary value problems for different hyperbolic systems have been studied in [2] extensively. What is different about our work here is that in addition to prescribing data on the characteristics, we also assume the extra condition (1.6) about $u$ on the $x$-axis.

2. The first-order system. We change the second-order system (1.4) to a first-order system by introducing

$$
u=\left[\begin{array}{l}
u_{1} \\
u_{2}
\end{array}\right], \quad u_{i t}=v_{i}, \quad u_{i x}=v_{i+2}, \quad i=1,2 .
$$

System (1.4) becomes

$$
\left[\begin{array}{cc}
0_{2} & -I_{2} \\
A & 0_{2}
\end{array}\right] v_{t}+\left[\begin{array}{cc}
I_{2} & 0_{2} \\
O_{2} & C
\end{array}\right] v_{x}=\left[\begin{array}{l}
0 \\
f
\end{array}\right]
$$

where $\mathrm{O}_{2}$ and $I_{2}$ are $2 \times 2$ zero and identity matrices. Multiply (2.2) by $\left[\begin{array}{cc}I_{2} & 0_{2} \\ 0_{2} & C\end{array}\right]^{-1}$ to obtain

$$
\left[\begin{array}{cc}
0_{2} & -I_{2} \\
C^{-1} A & 0_{2}
\end{array}\right] v_{t}+\left[\begin{array}{cc}
I_{2} & 0_{2} \\
O_{2} & I_{2}
\end{array}\right] v_{x}=\left[\begin{array}{ll}
I_{2} & 0_{2} \\
O_{2} & C
\end{array}\right]^{-1}\left[\begin{array}{l}
0 \\
f
\end{array}\right] .
$$

We rewrite system (2.3) in the form

$$
\tilde{A} v_{t}+v_{x}=F
$$

where

$$
\tilde{A}=\left[\begin{array}{cc}
0_{2} & -I_{2} \\
C^{-1} A & 0_{2}
\end{array}\right], \quad F=\left[\begin{array}{ll}
I_{2} & 0_{2} \\
0_{2} & C
\end{array}\right]^{-1}\left[\begin{array}{l}
0 \\
f
\end{array}\right]=\left[\begin{array}{c}
0 \\
C^{-1} f
\end{array}\right] .
$$

Based on our assumption on the form of the matrix $C^{-1} A$ in (1.8), we note that the eigenvalues of the matrix $\tilde{A}$ are \pm 1 and $\pm r$. Let $K$ be the matrix whose columns are the eigenvectors $k_{i}, i=1, \ldots, 4$, corresponding to the eigenvalues $-1,1,-r, r$, respectively. Making the change of variables $v=K w$, we obtain

$$
\tilde{A} K w_{t}+K w_{x}=F
$$


where $K$ is the matrix

$$
K=\left[\begin{array}{cccc}
-\frac{1+d}{c} & \frac{1+d}{c} & -\frac{d+r^{2}}{c r} & \frac{d+r^{2}}{c r} \\
1 & -1 & \frac{1}{r} & -\frac{1}{r} \\
-\frac{1+d}{c} & -\frac{1+d}{c} & -\frac{d+r^{2}}{c} & -\frac{d+r^{2}}{c} \\
1 & 1 & 1 & 1
\end{array}\right]
$$

Multiplying (2.6) by $K^{-1}$, we obtain

$$
\Lambda w_{t}+w_{x}=K^{-1} F
$$

where $K^{-1}$ is

$$
K^{-1}=\frac{1}{-2+2 r^{2}}\left[\begin{array}{cccc}
c & d+r^{2} & c & d+r^{2} \\
c & d+r^{2} & c & d+r^{2} \\
c r & (1+d) r & c & 1+d \\
c r & (1+d) r & c & 1+d
\end{array}\right]
$$

and $\Lambda$ is a $4 \times 4$ diagonal matrix with numbers $-1,1,-r, r$ on its main diagonal. Letting $w_{i}, F_{i}, i=1, \ldots, 4$, be the components of the vectors $w, F$ and noting that $F_{1}=F_{2}=0$ and $K^{-1} F$ is of the form

$$
K^{-1} F=\left[\begin{array}{c}
c_{1} F_{3}+c_{2} F_{4} \\
c_{1} F_{3}+c_{2} F_{4} \\
-c_{1} F_{3}+c_{3} F_{4} \\
-c_{1} F_{3}+c_{3} F_{4}
\end{array}\right]
$$

where

$$
c_{1}=\frac{c}{-2+2 r^{2}}, \quad c_{2}=\frac{d+r^{2}}{-2+2 r^{2}}, \quad c_{3}=\frac{1+d}{2-2 r^{2}},
$$

system (2.8) will be

$$
\begin{aligned}
-w_{1 t}+w_{1 x} & =c_{1} F_{3}+c_{2} F_{4}, \\
w_{2 t}+w_{2 x} & =c_{1} F_{3}+c_{2} F_{4}, \\
-r w_{3 t}+w_{3 x} & =-c_{1} F_{3}+c_{3} F_{4}, \\
r w_{4 t}+w_{4 x} & =-c_{1} F_{3}+c_{3} F_{4} .
\end{aligned}
$$

We solve system (2.12), (2.13), (2.14), and (2.15), in the triangle $T$, next.

3. The solution of the system. Consider (2.12). Take two points $P$ and $Q$ in the triangle $T$, along the characteristic $d x / d t=-1$, and integrate along the segment $P Q$ in the direction of the vector $\langle-1,1\rangle$. Let the arc length be $s$, then

$$
w_{1}(P)-w_{1}(Q)=\frac{1}{\sqrt{2}} \int_{P Q}\left(c_{1} F_{3}+c_{2} F_{4}\right) d s .
$$


Integrate (2.13) in $T$ from point $P$ to point $P_{1}$ along the characteristic $d x / d t=1$, in the direction of the vector $\langle 1,1\rangle$. We have

$$
w_{2}(P)-w_{2}\left(P_{1}\right)=-\frac{1}{\sqrt{2}} \int_{P P_{1}}\left(c_{1} F_{3}+c_{2} F_{4}\right) d s
$$

Integrate (2.14) from point $P$ to point $R$ in $T$ along the characteristic $d t / d x=-r$, in the direction of the vector $\langle-1, r\rangle$,

$$
w_{3}(P)-w_{3}(R)=\frac{1}{\sqrt{1+r^{2}}} \int_{P R}\left(-c_{1} F_{3}+c_{3} F_{4}\right) d s
$$

Similarly, if we integrate (2.15) from point $P$ to point $R_{1}$ in $T$ along the characteristic $d t / d x=r$, in the direction of $\langle 1, r\rangle$, we have

$$
w_{4}(P)-w_{4}\left(R_{1}\right)=-\frac{1}{\sqrt{1+r^{2}}} \int_{P R_{1}}\left(-c_{1} F_{3}+c_{3} F_{4}\right) d s
$$

Recall that $v=K w$ and $v=\left[u_{1 t}, u_{2 t}, u_{1 x}, u_{2 t}\right]^{t r}$. Then, (3.1), (3.2), (3.3), and (3.4) will become

$$
\begin{gathered}
\left.\left(c_{1}\left(u_{1 t}+u_{1 x}\right)+c_{2}\left(u_{2 t}+u_{2 x}\right)\right)\right|_{Q} ^{P}=\frac{1}{\sqrt{2}} \int_{P Q}\left(c_{1} F_{3}+c_{2} F_{4}\right) d s, \\
\left.\left(-c_{1}\left(u_{1 t}-u_{1 x}\right)-c_{2}\left(u_{2 t}-u_{2 x}\right)\right)\right|_{P_{1}} ^{P}=-\frac{1}{\sqrt{2}} \int_{P P_{1}}\left(c_{1} F_{3}+c_{2} F_{4}\right) d s, \\
\left.\left(-c_{1}\left(r u_{1 t}+u_{1 x}\right)+c_{3}\left(r u_{2 t}+u_{2 x}\right)\right)\right|_{R} ^{P}=\frac{1}{\sqrt{1+r^{2}}} \int_{P R}\left(-c_{1} F_{3}+c_{3} F_{4}\right) d s, \\
\left.\left(c_{1}\left(r u_{1 t}-u_{1 x}\right)-c_{3}\left(r u_{2 t}-u_{2 x}\right)\right)\right|_{R_{1}} ^{P}=-\frac{1}{\sqrt{1+r^{2}}} \int_{P R_{1}}\left(-c_{1} F_{3}+c_{3} F_{4}\right) d s .
\end{gathered}
$$

Now, take (3.5) and integrate it along the characteristic $d t / d x=1$ in the direction of the vector $\langle-1,-1\rangle$, so that the parallelogram $P Q Q_{2} Q_{1}$ inside $T$ is completed. We choose the vertex $Q$ on characteristic boundary $t=r x+1-r$, and $Q_{1}$ on the $x$-axis, then

$$
\begin{aligned}
c_{1} u_{1}(P)+c_{2} u_{2}(P)= & c_{1} u_{1}(Q)+c_{2} u_{2}(Q)-c_{1} u_{1}\left(Q_{2}\right)-c_{2} u_{2}\left(Q_{2}\right) \\
& +\int_{P Q_{2} Q_{1}}\left(c_{1} F_{3}+c_{2} F_{4}\right) d x d t .
\end{aligned}
$$

Note here that since we assumed $u=0$ along the $x$-axis, there are no terms involving $c_{1} u_{1}\left(Q_{1}\right)+c_{2} u_{2}\left(Q_{1}\right)$. Also, (3.9) gives a relationship between the values of the expression $c_{1} u_{1}+c_{2} u_{2}$ at two points $P$ and $Q_{2}$ inside $T$ and point $Q$ on the characteristic boundary, where points $P, Q, Q_{2}$, and $Q_{1}$ are vertices of a parallelogram inside $T$ with sides along the characteristics $d t / d x= \pm 1$. As it turns out, (3.6) will provide the same result if we put the point $P_{1}$ on the characteristic boundary $t=-r x+1+r$ and integrate along the characteristic $d t / d x=-1$ in the direction of the vector $\langle 1,-1\rangle$. In this 
case, we complete the parallelogram $P P_{1} P_{2} P_{3}$ with vertices $P$ and $P_{2}$ in the interior of $T$ and $P_{3}$ on the $x$-axis, that is,

$$
\begin{aligned}
c_{1} u_{1}(P)+c_{2} u_{2}(P)= & c_{1} u_{1}\left(P_{1}\right)+c_{2} u_{2}\left(P_{1}\right)-c_{1} u_{1}\left(P_{2}\right)-c_{2} u_{2}\left(P_{2}\right) \\
& +\int_{P P_{1} P_{2} P_{3}}\left(c_{1} F_{3}+c_{2} F_{4}\right) d x d t .
\end{aligned}
$$

In (3.7), if we put the point $R$ on a characteristic boundary $t=r x+1-r$ and integrate along the characteristic $d t / d x=r$ in the direction of the vector $\langle-1,-r\rangle$ so that the parallelogram $P R M P_{1}$ in $T$ is completed with $P_{1}$ on the $x$-axis, $R$ and $M$ on $t=r x+1-r$, and $P$ in $T$, we have

$$
\begin{aligned}
-c_{1} u_{1}(P)+c_{3} u_{2}(P)= & -c_{1} u_{1}(R)+c_{3} u_{2}(R)+c_{1} u_{1}(M)-c_{3} u_{2}(M) \\
& +\int_{P R M P_{1}}\left(-c_{1} F_{3}+c_{3} F_{4}\right) d x d t .
\end{aligned}
$$

The integration of (3.8) in the direction $\langle 1,-r\rangle$ will result in the same equation as in (3.10), that is,

$$
\begin{aligned}
-c_{1} u_{1}(P)+c_{3} u_{2}(P)= & -c_{1} u_{1}\left(R_{1}\right)+c_{3} u_{2}\left(R_{1}\right)+c_{1} u_{1}\left(R_{2}\right)-c_{3} u_{2}\left(R_{2}\right) \\
& +\int_{P R_{1} R_{2} R_{3}}\left(-c_{1} F_{3}+c_{3} F_{4}\right) d x d t,
\end{aligned}
$$

where $P$ is in $T, R_{1}$ and $R_{2}$ are on $t=-r x+1+r$, and $R_{3}$ is on the $x$-axis. Now, we are in a position to apply condition (1.7). Write (3.11) for the parallelogram whose side $P R$ meets the characteristic boundary $t=-r x+1+r$ and the side $R M$ is on the characteristic boundary $t=r x+1-r$. Denoting the vertex $(0,0)$ of $T$ by $O,(3.11)$ for the parallelogram $P^{\prime} O M P_{1}$ becomes

$$
\begin{aligned}
-c_{1} u_{1}\left(P^{\prime}\right)+c_{3} u_{2}\left(P^{\prime}\right)= & -c_{1} u_{1}(O)+c_{3} u_{2}(O)+c_{1} u_{1}(M)-c_{3} u_{2}(M) \\
& +\int_{P^{\prime} O M P_{1}}\left(-c_{1} F_{3}+c_{3} F_{4}\right) d x d t
\end{aligned}
$$

Since $u\left(P^{\prime}\right)=u(M)$ and $u(O)=0,(3.13)$ yields

$$
c_{1} u_{1}(M)-c_{3} u_{2}(M)=-\frac{1}{2} \int_{P^{\prime} O M P_{1}}\left(-c_{1} F_{3}+c_{3} F_{4}\right) d x d t .
$$

This time, write (3.12) for the parallelogram $P^{\prime \prime} R O M^{\prime}$, where the side $O R$ is along the characteristic boundary $t=r x+1-r$, the side $O M^{\prime}$ is on the characteristic boundary $t=-r x+1+r$, and the point $P^{\prime \prime}$ is on the $x$-axis:

$$
\begin{aligned}
-c_{1} u_{1}(R)+c_{3} u_{2}(R)= & -c_{1} u_{1}(O)+c_{3} u_{2}(O)+c_{1} u_{1}\left(M^{\prime}\right)-c_{3} u_{2}\left(M^{\prime}\right) \\
& +\int_{P^{\prime \prime} R O M^{\prime}}\left(-c_{1} F_{3}+c_{3} F_{4}\right) d x d t
\end{aligned}
$$

which, upon using $u\left(M^{\prime}\right)=u(R)$ and $u(O)=0$, yields

$$
-c_{1} u_{1}(R)+c_{3} u_{2}(R)=\frac{1}{2} \int_{P^{\prime \prime} R O M^{\prime}}\left(-c_{1} F_{3}+c_{3} F_{4}\right) d x d t
$$




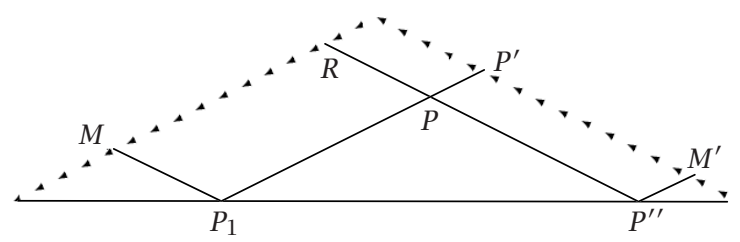

FIGURE 3.1. The regions in the definition of $G(P ; x, t)$.

From (3.11), (3.14), and (3.15), we obtain

$$
\begin{aligned}
-c_{1} u_{1}(P)+c_{3} u_{2}(P)= & \frac{1}{2} \int_{P^{\prime \prime} R O M^{\prime}}\left(-c_{1} F_{3}+c_{3} F_{4}\right) d x d t \\
& -\frac{1}{2} \int_{P^{\prime} O M P_{1}}\left(-c_{1} F_{3}+c_{3} F_{4}\right) d x d t \\
& +\int_{P R M P_{1}}\left(-c_{1} F_{3}+c_{3} F_{4}\right) d x d t
\end{aligned}
$$

Equation (3.17) can be put in the compact form

$$
-c_{1} u_{1}(P)+c_{3} u_{2}(P)=\iint_{T} G(P ; x, t)\left(-c_{1} F_{3}+c_{3} F_{4}\right) d x d t
$$

where $G$ is Green's function with values described as follows. Fix a point $P$ in the triangular region $T$ bounded by $t=0$ and characteristics $t=r x+1-r$ and $t=-r x+1+r$. From $P$, draw lines parallel to these characteristics so that one line meets the side $t=-r x+1+r$ at $P^{\prime}$ and $t=0$ at $P_{1}$. The other line meets $t=r x+1-r$ at $R$ and $t=0$ at $P^{\prime \prime}$. From $P_{1}$, draw a line parallel to $t=-r x+1+r$ to meet the line $t=r x+1-r$ at $M$. From $P^{\prime \prime}$, draw a line parallel to $t=r x+1-r$ to meet $t=-r x+1+r$ at $M^{\prime}$. Then $G$ is defined by

$$
G(P ; x, t)= \begin{cases}\frac{1}{2}, & (x, t) \in P R M P_{1} \cup P P^{\prime} M^{\prime} P^{\prime \prime}, \\ 0, & (x, t) \in T \backslash\left(P R M P_{1} \cup P P^{\prime} M^{\prime} P^{\prime \prime}\right)\end{cases}
$$

see Figure 3.1.

Now we consider (3.9) and (3.10). From either one of these equations, we can calculate the value of $c_{1} u_{1}(P)+c_{2} u_{2}(P)$ by using the data $g$ given in condition (1.7). For instance, using (3.10) with $P$ and $Q_{0}$ in $T, R_{0}$ on $t=-r x+1+r$, and $S_{0}$ on the $x$-axis, we have

$$
\begin{aligned}
c_{1} u_{1}(P)+c_{2} u_{2}(P)= & c_{1} u_{1}\left(R_{0}\right)+c_{2} u_{2}\left(R_{0}\right)-c_{1} u_{1}\left(Q_{0}\right)-c_{2} u_{2}\left(Q_{0}\right) \\
& +\int_{P R_{0} Q_{0} S_{0}}\left(c_{1} F_{3}+c_{2} F_{4}\right) d x d t .
\end{aligned}
$$

For convenience, we denote

$$
\alpha=c_{1} u_{1}+c_{2} u_{2}
$$


In terms of notation (3.21), we have

$$
\alpha(P)=\alpha\left(R_{0}\right)-\alpha\left(Q_{0}\right)+\int_{P R_{0} Q_{0} S_{0}}\left(c_{1} F_{3}+c_{2} F_{4}\right) d x d t
$$

But then, using (3.22) again, this time starting at the point $Q_{0}$ and completing the parallelogram $Q_{0} R_{1} Q_{1} S_{1}$, we have

$$
\alpha\left(Q_{0}\right)=\alpha\left(R_{1}\right)-\alpha\left(Q_{1}\right)+\int_{Q_{0} R_{1} Q_{1} S_{1}}\left(c_{1} F_{3}+c_{2} F_{4}\right) d x d t .
$$

We substitute $\alpha\left(Q_{0}\right)$ from (3.23) into (3.22) to obtain

$$
\begin{aligned}
\alpha(P)= & \alpha\left(R_{0}\right)-\alpha\left(R_{1}\right)+\alpha\left(Q_{1}\right)-\int_{Q_{0} R_{1} Q_{1} S_{1}}\left(c_{1} F_{3}+c_{2} F_{4}\right) d x d t \\
& +\int_{P R_{0} Q_{0} S_{0}}\left(c_{1} F_{3}+c_{2} F_{4}\right) d x d t .
\end{aligned}
$$

Continuing this process and writing (3.22) for the points $Q_{1}, Q_{2}, \ldots, Q_{n}, n=0,1,2, \ldots$, we obtain the equation

$$
\begin{aligned}
\alpha(P)= & \sum_{n=0}^{m}(-1)^{n} \alpha\left(R_{n}\right)+\sum_{n=0}^{m}(-1)^{n} \iint_{Q_{n-1} R_{n} Q_{n} S_{n}}\left(c_{1} F_{3}+c_{2} F_{4}\right) d x d t \\
& +(-1)^{m+1} \alpha\left(Q_{m}\right),
\end{aligned}
$$

where $Q_{-1}=P$. The parallelograms $Q_{n-1} R_{n} Q_{n} S_{n}, n=0, \ldots, m$, have vertices $R_{0}, R_{1}, \ldots$, $R_{m}$, on the characteristic boundary moving toward the point $(1+1 / r, 0)$. The points $Q_{1}, Q_{2}, \ldots, Q_{m+1}$ are the vertices opposite $P$ in $T$ and $S_{0}, S_{1}, \ldots, S_{m}$ are on the $x$-axis. Now, we take the limit of (3.25) as $m \rightarrow \infty$ :

$$
\begin{aligned}
\lim _{m \rightarrow \infty} \alpha(P)= & \sum_{n=0}^{\infty}(-1)^{n} \alpha\left(R_{n}\right)+\lim _{m \rightarrow \infty}(-1)^{m+1} \alpha\left(Q_{m}\right) \\
& +\sum_{n=0}^{\infty}(-1)^{n} \iint_{Q_{n-1} R_{n} Q_{n} S_{n}}\left(c_{1} F_{3}+c_{2} F_{4}\right) d x d t
\end{aligned}
$$

and note that in this process, the points $R_{m}, Q_{m}$, and $S_{m}$ all approach the point (1+ $1 / r, 0)$ in $T$. Since data is zero at this point by condition $(1.7)$, we must have

$$
\lim _{m \rightarrow \infty}(-1)^{m+1} \alpha\left(Q_{m}\right)=0
$$

The series involving the integrals over the parallelograms converges because the union of all these parallelograms is still a subset of the region $T$ and the function $c_{1} F_{3}+c_{2} F_{4}$ is integrable over $T$, being continuous there. It now remains to ascertain the convergence of $\sum_{n=0}^{\infty}(-1)^{n} \alpha\left(R_{n}\right)$. For this purpose, we use the assumption, in condition (1.7), that the components $g_{1}$ and $g_{2}$ of the data function $g$ along the characteristics are monotonically decreasing to zero in the set $\left[0, t_{0}\right] \cup\left[t_{1}, 1\right], t_{0}<t_{1}$ for some $t_{0}, t_{1}$ in $(0,1)$. Then, the infinite sum $\sum_{n=0}^{\infty}(-1)^{n} \alpha\left(R_{n}\right)$ converges because it is a monotonically decreasing alternating series with $\lim _{n \rightarrow \infty} \alpha\left(R_{n}\right)=0$. Since the positions of the points $R_{n}$ on the 


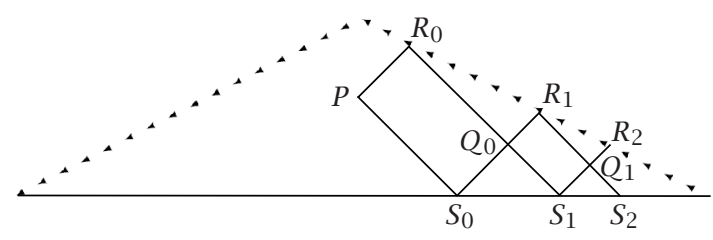

FIGURE 3.2. The regions in the definition of $H(P ; x, t)$.

characteristics depend on the point $P$, we show this dependence by $R_{n}=R_{n}(r, P)$ and write

$$
\sum_{n=0}^{\infty}(-1)^{n} \alpha\left(R_{n}(r, P)\right)=c_{1} h_{1}(r ; P)+c_{2} h_{2}(r ; P),
$$

where $h_{1}$ and $h_{2}$ are the limiting functions of the series $\sum_{n=0}^{\infty}(-1)^{n} u_{1}\left(R_{n}\right)$ and $\sum_{n=0}^{\infty}$ $(-1)^{n} u_{2}\left(R_{n}\right)$, respectively. If we rewrite (3.25) in terms of $u_{1}, u_{2}$ and use (3.27) and (3.28), we have

$$
\begin{aligned}
c_{1} u_{1}(P)+c_{2} u_{2}(P)= & c_{1} h_{1}(r ; P)+c_{2} h_{2}(r ; P) \\
& +\sum_{n=0}^{\infty}(-1)^{n} \iint_{Q_{n-1} R_{n} Q_{n} S_{n}}\left(c_{1} F_{3}+c_{2} F_{4}\right) d x d t .
\end{aligned}
$$

We can rewrite (3.29) as follows:

$$
\begin{aligned}
c_{1} u_{1}(P)+c_{2} u_{2}(P)= & c_{1} h_{1}(r ; P)+c_{2} h_{2}(r ; P) \\
& +\iint_{T} H(P ; x, t)\left(c_{1} F_{3}+c_{2} F_{4}\right) d x d t,
\end{aligned}
$$

where

$$
H(P ; x, t)= \begin{cases}(-1)^{n}, & (x, t) \in Q_{n-1} R_{n} Q_{n} S_{n}, \quad n=0,1, \ldots \\ 0, & (x, t) \in T \backslash \cup_{0}^{\infty} Q_{n-1} R_{n} Q_{n} S_{n}\end{cases}
$$

see Figure 3.2.

Putting (3.18) and (3.31) together, we can write

$$
\begin{aligned}
{\left[\begin{array}{cc}
c_{1} & c_{2} \\
-c_{1} & c_{3}
\end{array}\right]\left[\begin{array}{l}
u_{1}(P) \\
u_{2}(P)
\end{array}\right]=} & {\left[\begin{array}{cc}
c_{1} & c_{2} \\
0 & 0
\end{array}\right]\left[\begin{array}{l}
h_{1}(r ; P) \\
h_{2}(r ; P)
\end{array}\right] } \\
& +\iint_{T}\left[\begin{array}{cc}
H & 0 \\
0 & G
\end{array}\right]\left[\begin{array}{cc}
c_{1} & c_{2} \\
-c_{1} & c_{3}
\end{array}\right]\left[\begin{array}{l}
F_{3} \\
F_{4}
\end{array}\right] d x d t
\end{aligned}
$$

Recall that $\left[\begin{array}{c}F_{3} \\ F_{4}\end{array}\right]=C^{-1} f$. Then, (3.32) can be rewritten in the form

$$
u(P)=L h(P)+\iint_{T} N(P ; x, t) f(x, t) d x d t, \quad P \in T,
$$


where $h=\left[\begin{array}{l}h_{1} \\ h_{2}\end{array}\right]$ for $h_{1}, h_{2}$ are as defined in (3.28), $L=\left[\begin{array}{cc}c_{1} & c_{2} \\ -c_{1} & c_{3}\end{array}\right]^{-1}\left[\begin{array}{cc}c_{1} & c_{2} \\ 0 & 0\end{array}\right]$, and $N=$ $C^{-1}\left[\begin{array}{ll}H & 0 \\ 0 & G\end{array}\right]$. Equation (3.33) provides a unique solution to problem (1.4), (1.5), (1.6), and (1.7) in $C^{2}(T)$. It is unique by the way it has been obtained. Therefore, we have the following theorem.

THEOREM 3.1. Let $f(x, t)$ with values in $\mathbb{R}^{2}$ be a continuous function in $T$, and let $g(r, t)$, also with values in $\mathbb{R}^{2}$, be $C^{2}$ in $t$ for $t \in[0,1]$, for any $r \in(0,1)$, and components $g_{1}$ and $g_{2}$ that vanish monotonically to zero as $t$ goes to zero or one in $\left[0, t_{0}\right] \cup\left[t_{1}, 1\right]$ for some $t_{0}<t_{1}, t_{0}, t_{1} \in(0,1)$. Let (1.4) be a strictly hyperbolic $2 \times 2$ system with constant matrices $A$ and $C$ satisfying $\operatorname{det} C \neq 0$ and condition (1.8). Then, the boundary value problem (1.4), (1.5), (1.6), and (1.7) has a unique solution of the form (3.33) in $C^{2}(T)$.

\section{REFERENCES}

[1] T. Sh. Kal'menov, The spectrum of a selfadjoint problem for the wave equation, Vestnik Akad. Nauk Kazakh. SSR (1983), no. 1, 63-66.

[2] S. Kharibegashvili, Goursat and Darboux type problems for linear hyperbolic partial differential equations and systems, Mem. Differential Equations Math. Phys. 4 (1995), 1-127.

[3] K. Kreith, Symmetric Green's function for a class of CIV boundary value problems, Canad. Math. Bull. 31 (1988), no. 3, 272-279.

Nezam Iraniparast: Department of Mathematics, Western Kentucky University, Bowling Green, KY 42101-3576, USA

E-mail address: nezam.i raniparast@wku.edu 


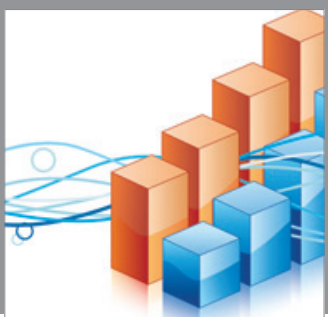

Advances in

Operations Research

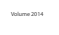

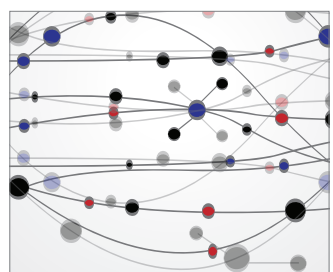

\section{The Scientific} World Journal
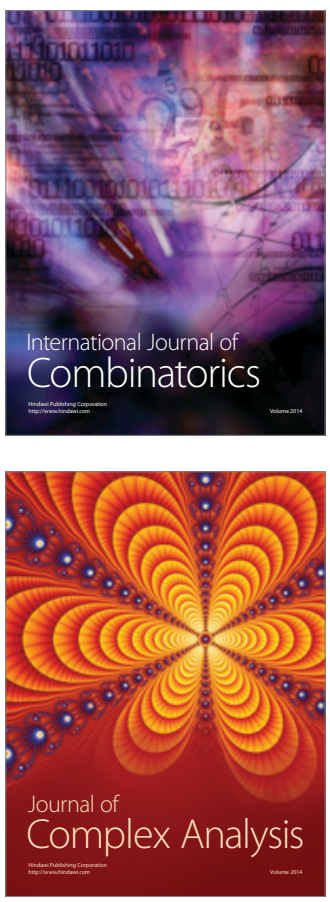

International Journal of

Mathematics and

Mathematical

Sciences
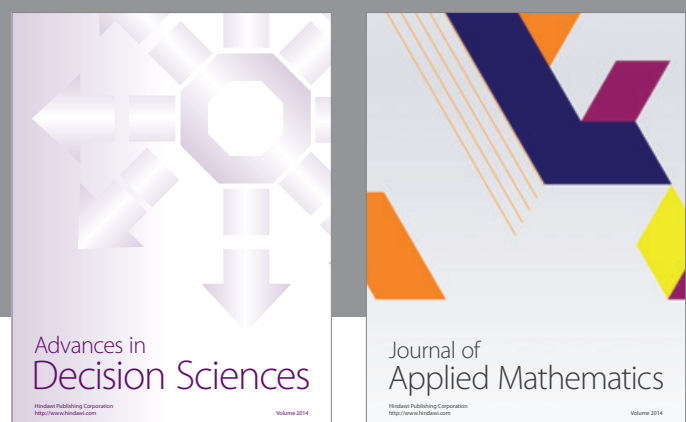

Journal of

Applied Mathematics
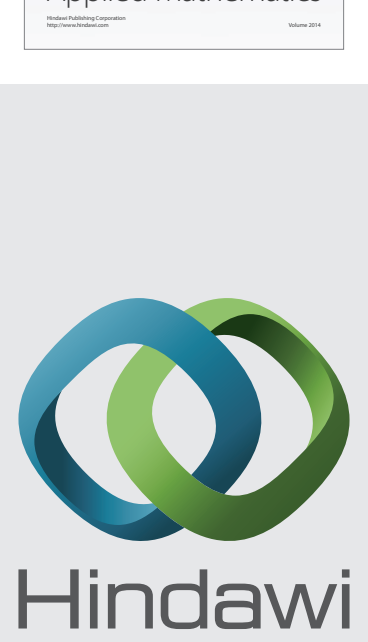

Submit your manuscripts at http://www.hindawi.com
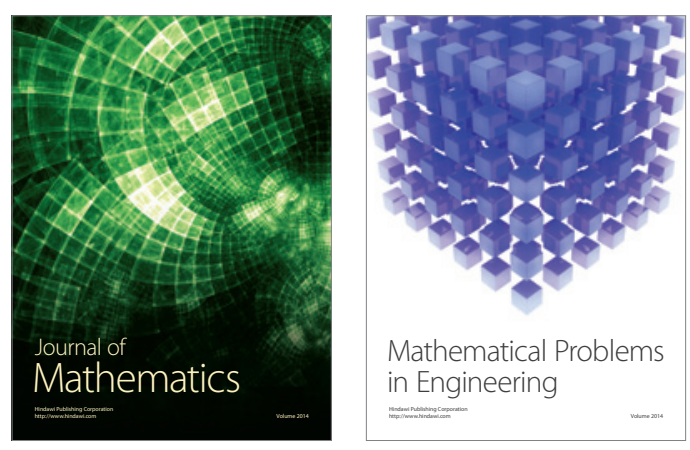

Mathematical Problems in Engineering
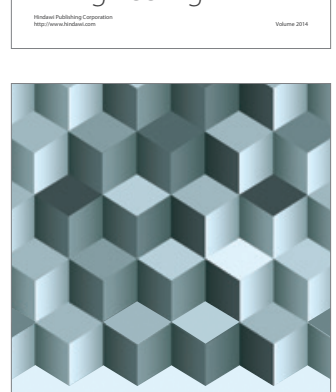

Journal of

Function Spaces
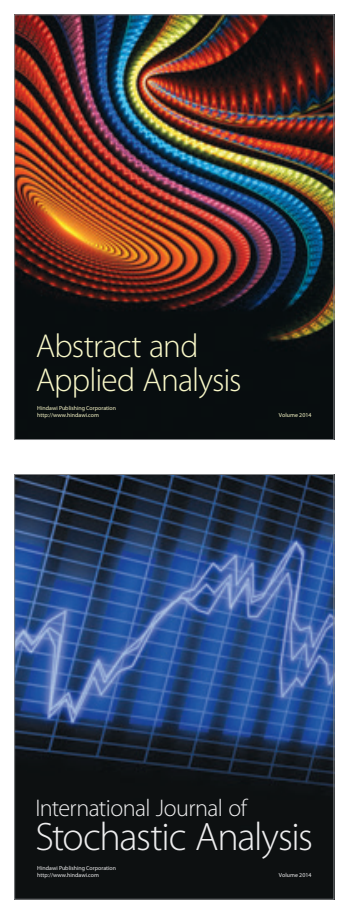

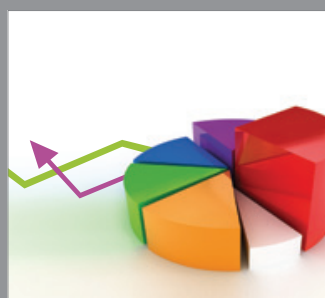

ournal of

Probability and Statistics

Promensencen
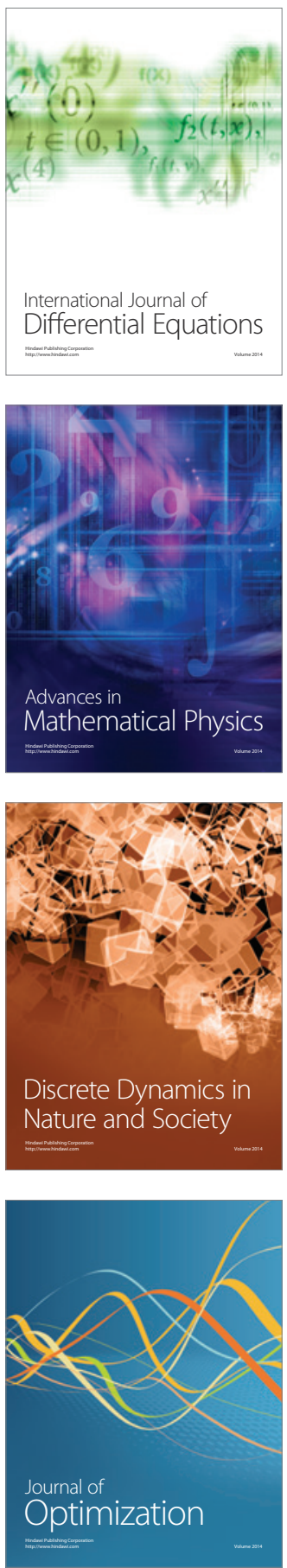